\title{
New Low-mass Accretors in the Scorpius-Centaurus OB Association
}

\author{
Simon J. Murphy ${ }^{1,2}$, Warrick A. Lawson ${ }^{2}$ and Joao Bento ${ }^{1}$ \\ ${ }^{1}$ Research School of Astronomy \& Astrophysics, Australian National Unversity \\ email: simon.murphy@anu.edu.au \\ ${ }^{2}$ School of Physical, Environmental and Mathematical Sciences, \\ University of New South Wales Canberra
}

\begin{abstract}
We describe the serendipitous discovery of two new lithium-rich M5 members of the Scorpius-Centaurus OB Association (Sco-Cen). Both stars exhibit large 12 and $22 \mu \mathrm{m}$ excesses and strong, variable $\mathrm{H} \alpha$ emission which we attribute to accretion from circumstellar discs. Such stars are thought to be incredibly rare at the $\sim 16$ Myr median age of much of Sco-Cen. The serendipitous discovery of two accreting stars hosting large quantities of circumstellar material may be indicative of a sizeable age spread in Sco-Cen, or further evidence that disc dispersal and planet formation time-scales are longer around lower-mass stars.
\end{abstract}

Keywords. circumstellar matter, open clusters and associations: individual (Sco-Cen), stars: low-mass, stars: pre-main sequence

\section{Introduction}

Nearby stars with ages of $<20 \mathrm{Myr}$ are ideal laboratories in which to investigate the end stages of star and planet formation. It is over this time period that gas-rich protoplanetary discs which feed giant planet formation and circumstellar accretion dissipate and are replaced by gas-poor, dusty debris discs (Wyatt 2008; Williams \& Cieza 2011).

The Scorpius-Centaurus OB Association (Sco-Cen, Sco OB2; Blaauw 1964; de Zeeuw et al. 1999; Preibisch \& Mamajek 2008) is the closest site of recent massive star formation and the dominant population of pre-main sequence stars in the solar neighbourhood. ScoCen has traditionally been divided into three subgroups, each with subtly distinct mean distances, ages and space motions: Upper Scorpius (145 pc), Upper Centaurus Lupus (UCL, 140 pc) and Lower Centaurus Crux (LCC 120 pc; de Zeeuw et al. 1999). The subgroups have median ages of approximately $10 \mathrm{Myr}, 16 \mathrm{Myr}$ and $17 \mathrm{Myr}$, respectively (Mamajek et al. 2002; Pecaut et al. 2012). While the low-mass population of Upper Scorpius has been well-studied in recent years, little is known of the older and larger UCL and LCC subgroups below $1 M_{\odot}$. In this contribution we describe the serendipitous discovery of two rare accreting M5 stars in LCC ( 2MASS J12392312-5702400 ) and UCL ( 2MASS J14224891-3623009 ), found during a search for new members of the Octans Association. Further information on the candidate selection procedures, spectroscopic observations and new members of the 10 Myr-old TW Hydrae Association also identified during this work can be found in Murphy et al. (2015, submitted).

\section{Membership in Sco-Cen}

2M1239-5702 and 2M1422-3623 lie within the classical boundaries of LCC and UCL, respectively, with SPM4 proper motions similar to higher-mass members identified with Hipparcos (de Zeeuw et al. 1999). These proper motions correspond to kinematic 

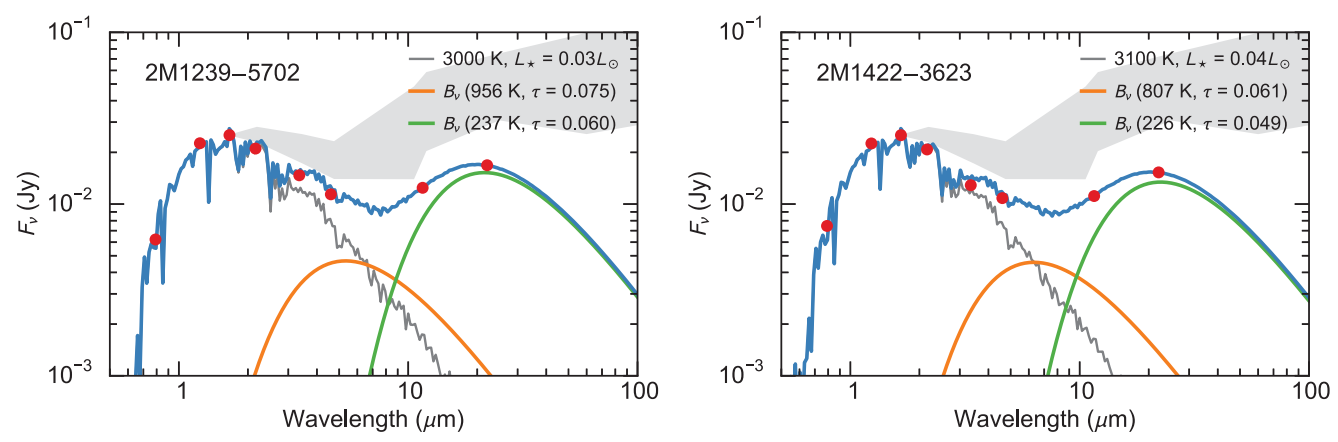

Figure 1. SEDs of 2M1239-5702 (left) and 2M1422-3623 (right). Photometry from DENIS, 2MASS and WISE is plotted, with a solar-metallicity BT-Settl model fitted to the $\lambda<3 \mu \mathrm{m}$ data. The WISE excesses are fit using blackbodies of the specified temperature and fractional luminosity $\left(\tau=L_{I R} / L_{\star}\right)$. The shaded region is the interquartile range of Class I K5-M2 sources observed in Taurus from D'Alessio et al. (1999), normalised at $H(1.6 \mu \mathrm{m})$.

distances of $119_{-11}^{+13} \mathrm{pc}$ and $142_{-24}^{+35} \mathrm{pc}$, assuming the mean LCC and UCL space motions of Chen et al. (2011), and are in excellent agreement with the mean Hipparcos distances of the subgroups. At these distances $2 \mathrm{M} 1239-5702$ and $2 \mathrm{M} 1422-3623$ are $3 \mathrm{~km} \mathrm{~s}^{-1}$ and $6 \mathrm{~km} \mathrm{~s}^{-1}$, respectively, from the Chen et al. mean space motions, with uncertainties of $2-4 \mathrm{~km} \mathrm{~s}^{-1}$ in each velocity component. This is well within the scatter of higher-mass Sco-Cen members with known radial velocities (Murphy et al. 2015, submitted). Their colour-magnitude diagram (CMD) positions suggest an age of approximately $10 \mathrm{Myr}$, similar to TW Hydrae members and consistent with recent age ranges for UCL and LCC (Song et al. 2012; Pecaut et al. 2012). 2M1422-3623 is $15-20^{\circ}$ from the Lupus dark clouds within UCL. Given its large separation from confirmed Lupus sources (Galli et al. 2013), we do not believe it is a member of this much younger (age $<5 \mathrm{Myr}$ ) star-forming region. 2M1239-5702 lies near the centre of LCC, $\sim 1^{\circ}$ from the foreground M-giant $\gamma$ Cru.

\section{Disc and accretion properties}

2M1239-5702 and 2M1422-3623 were two of only a handful of spectroscopic candidates in our Octans survey to show excesses in 12 and $22 \mu \mathrm{m}$ Wide-field Infrared Survey Explorer (WISE; Wright et al. 2010) photometry. Their remarkably similar spectral energy distributions (SEDs) are plotted in Figure 1. Both are consistent with somewhat evolved, 'homologously-depleted' discs in the process of becoming optically thin, without strong evidence for the inner holes or gaps seen in 'transitional'-type objects (Espaillat et al. 2012). Double-temperature blackbody models with a cool $\sim 230 \mathrm{~K}$ outer component and a warmer 800-900 K inner component provide a good fit to the WISE photometry in both cases. Assuming $L_{*}=0.03 L_{\odot}$ from integration of the underlying photospheric flux (Figure 1) and large grains, these temperatures correspond to disc radii of $0.25 \mathrm{AU}$ and 0.02 AU, respectively (Backman \& Paresce 1993).

We also observed broad, variable $\mathrm{H} \alpha$ emission from both stars which exceeded the equivalent width (15-18 A; Fang et al. 2009) and velocity width at $10 \%$ flux $\left(270 \mathrm{~km} \mathrm{~s}^{-1}\right.$; White \& Basri 2003) criteria for confirming circumstellar disc accretion. Multi-epoch H $\alpha$ velocity profiles are plotted in Figure 2. Equivalent widths for 2M1422-3623 ranged from -91 to $-33 \AA$ over the four epochs of medium-resolution $(R=7,000)$ Australian National University $2.3-\mathrm{m} / \mathrm{WiFeS}$ spectroscopy, with $v_{10}$ velocity widths of $240-340 \mathrm{~km} \mathrm{~s}^{-1}$. For 2M1239-5702 these ranges were -63 to $-27 \AA$ and $240-330 \mathrm{~km} \mathrm{~s}^{-1}$ across two epochs. 


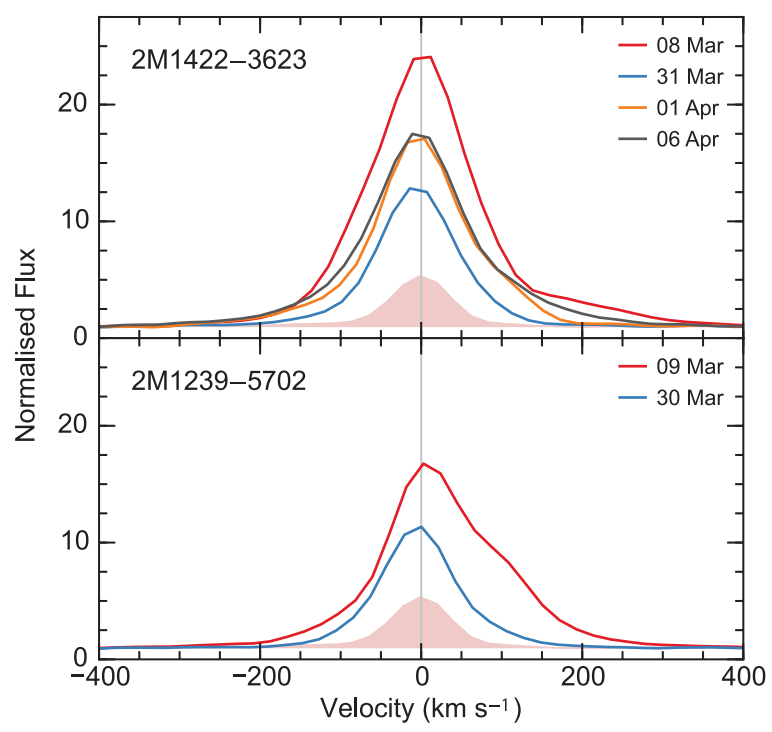

Figure 2. 2.3-m/WiFeS $\mathrm{H} \alpha$ velocity profiles for 2M1422-3623 (top) and 2M1239-5702 (bottom). Each panel also shows the emission line profile of a typical active but non-accreting M-dwarf of similar spectral type $\left(\mathrm{EW}=-9.5 \AA, v_{10}=169 \mathrm{~km} \mathrm{~s}^{-1}\right)$.

Applying the $v_{10}-\dot{M}$ relation of Natta et al. (2004) yields maximal mass accretion rates of $\sim 10^{-9.5} M_{\odot} \mathrm{yr}^{-1}$ with a variation of $\sim 1$ dex across epochs. 2M1239-5702 and 2M1422-3623 show near-IR excesses at 3-5 $\mathrm{m}$, indicating the presence of some dust in the inner disc. The variable accretion we observed is presumably driven by clumpy gas-rich material in this region.

In addition to $\mathrm{H} \alpha$ we also observed $\mathrm{Na}$ I $\mathrm{D}$ and forbidden [O I] $\lambda 6300 / 6363$ emission in 2M1422-3623 and strong He I $\lambda 5876 / 6678$ emission from both stars. The Na I and He I emission are believed to originate with $\mathrm{H} \alpha$ in magnetospheric infall regions (Muzerolle et al. 1998), whereas the forbidden emission is usually attributed to accretion-driven outflows or winds (Appenzeller \& Mundt 1989).

\section{Discussion}

Outside of the younger ( $\sim 10$ Myr) Upper Scorpius subgroup, 2M1239-5702 and 2M1422-3623 join a select group of Sco-Cen members known to be accreting. These include the M3.5 LCC member 2MASS J13373839-4736297 (Rodriguez et al. 2011) and the higher-mass accretors HD 139614, HD 135344 and AK Sco (Preibisch \& Mamajek 2008). The rarity of such objects is unsurprising given the observed rapid decline of accretor fraction with age, with $e$-folding time-scales of 2-3 Myr (e.g. Fedele et al. 2010). For a putative population of $10^{4}$ low-mass Sco-Cen members we may naively expect only a handful of active accretors to have survived until the $\sim 16$ Myr median age of UCL and LCC. The serendipitous discovery of two such objects in a survey for Octans members therefore seems very unlikely. We propose two scenarios which may explain the presence of these supposedly rare objects - significant age spreads in the Sco-Cen subgroups, and/or increased circumstellar disc lifetimes around lower-mass stars.

Mamajek et al. (2002) and Pecaut et al. (2012) have investigated the issue of intrinsic age spreads in UCL and LCC. They found $1 \sigma$ spreads of a few Myr up to 8 Myr depending on the subgroup, spectral type and evolutionary model grid considered. At face value, this 
would imply star formation in the subgroups ceased in the last 5-10 Myr, or even later if the larger age spreads are realistic. There is also evidence for spatial variation in ages within the subgroups (Pecaut \& Mamajek, in prep; Preibisch \& Mamajek 2008). Given these proposed age spreads, 2M1239-5702 and 2M1422-3623 may be as young as 5$10 \mathrm{Myr}$ and represent some of the last stars to have formed in UCL and LCC. Outside of the presence of substantial circumstellar material and accretion, young ages for both stars are supported by their CMD positions and undepleted lithium $\left(\mathrm{EW}_{\lambda 6708}>600 \mathrm{~mA}\right)$.

A second possibility is that the time-scale for circumstellar disc dispersal is stellar mass dependent, being less efficient around lower-mass stars. Such a trend has been observed in several young groups, including Upper Scorpius (Carpenter et al. 2006; Kennedy \& Kenyon 2009; Luhman \& Mamajek 2012) and has critical implications for the time available to form giant planets around low-mass stars. These studies indicate that a significant fraction $(\sim 25 \%)$ of M-dwarfs are able to retain inner discs at an age of $10 \mathrm{Myr}$. Extreme age spreads in Sco-Cen would thus not be required to explain the excesses and accretion observed in 2M1239-5702 and 2M1422-3623. If such a trend is universal there may be hundreds more disc-bearing UCL and LCC members (out of a total low-mass population of several thousand stars) awaiting discovery within WISE and contemporary proper motion catalogues (e.g. UCAC4, SPM4), and soon, Gaia. These new members would also be very useful for lithium depletion (e.g. Song et al. 2012) and exoplanet direct imaging studies, among other works.

\section{Acknowledgement}

SJM thanks the IAU and organisers of IAUS 314 for the generous award of a travel grant to attend the symposium and present this work.

\section{References}

Appenzeller I. \& Mundt R., 1989, ARAA, 1, 291

Backman D. E. \& Paresce F., 1993, in E.H. Levy, J.I. Lunine, eds, Protostars and Planets III. pp. $1253-1304$

Blaauw A., 1964, ARAA, 2, 213

Carpenter J. M., Mamajek E. E., Hillenbrand L. A., \& Meyer M. R., 2006, ApJL, 651, L49

Chen C. H., Mamajek E. E., Bitner M. A., Pecaut M., Su K. Y. L., \& Weinberger A. J., 2011, ApJ, 738, 122

D'Alessio P., Calvet N., Hartmann L., Lizano S., \& Cantó J., 1999, ApJ, 527, 893

de Zeeuw P. T., Hoogerwerf R., de Bruijne J. H. J., Brown A. G. A., \& Blaauw A., 1999, AJ, 117,354

Espaillat C. et al., 2012, ApJ, 747, 103

Fang M., van Boekel R., Wang W., Carmona A., Sicilia-Aguilar A., \& Henning T., 2009, A $\mathscr{J} A$, 504,461

Fedele D., van den Ancker M. E., Henning T., Jayawardhana R., \& Oliveira J. M., 2010, A\&্A, $510, \mathrm{~A} 72$

Galli P. A. B., Bertout C., Teixeira R., \& Ducourant C., 2013, A\& A, 558, A77

Kennedy G. M. \& Kenyon S. J., 2009, ApJ, 695, 1210

Luhman K. L. \& Mamajek E. E., 2012, ApJ, 758, 31

Mamajek E. E., Meyer M. R., Liebert J., 2002, AJ, 124, 1670

Muzerolle J., Hartmann L., \& Calvet N., 1998, AJ, 116, 455

Natta A., Testi L., Muzerolle J., Randich S., Comerón F., \& Persi P., 2004, A\&̈A, 424, 603

Pecaut M. J., Mamajek E. E., \& Bubar E. J., 2012, ApJ, 746, 154

Preibisch T. \& Mamajek E. E., 2008, Handbook of Star Forming Regions, Vol. II. The Southern Sky, ASP Press. p. 235

Rodriguez D. R., Bessell M. S., Zuckerman B., \& Kastner J. H., 2011, ApJ, 727, 62 
Song I., Zuckerman B., \& Bessell M. S., 2012, AJ, 144, 8

White R. J. \& Basri G., 2003, ApJ, 582, 1109

Williams J. P. \& Cieza L. A., 2011, ARAA, 49, 67

Wright E. L. et al., 2010, AJ, 140, 1868

Wyatt M. C., 2008, ARAA, 46, 339 